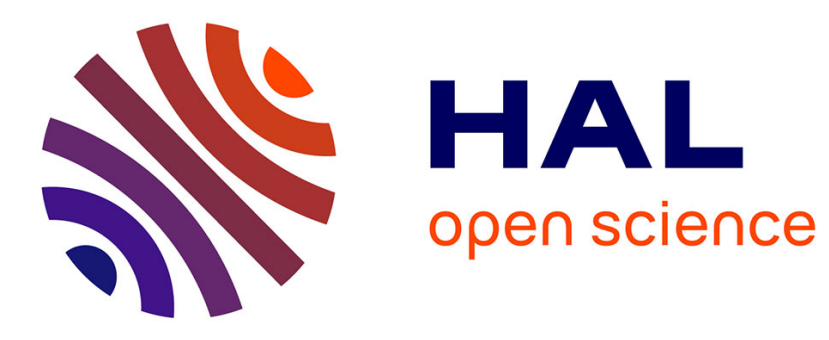

\title{
Interlayer correlations in smectic B phases
}

A.J. Leadbetter, J.C. Frost, M.A. Mazid

\section{To cite this version:}

A.J. Leadbetter, J.C. Frost, M.A. Mazid. Interlayer correlations in smectic B phases. Journal de Physique Lettres, 1979, 40 (14), pp.325-329. 10.1051/jphyslet:019790040014032500 . jpa-00231636

\section{HAL Id: jpa-00231636 https://hal.science/jpa-00231636}

Submitted on 1 Jan 1979

HAL is a multi-disciplinary open access archive for the deposit and dissemination of scientific research documents, whether they are published or not. The documents may come from teaching and research institutions in France or abroad, or from public or private research centers.
L'archive ouverte pluridisciplinaire HAL, est destinée au dépôt et à la diffusion de documents scientifiques de niveau recherche, publiés ou non, émanant des établissements d'enseignement et de recherche français ou étrangers, des laboratoires publics ou privés. 


\title{
Interlayer correlations in smectic B phases
}

\author{
A. J. Leadbetter, J. C. Frost and M. A. Mazid \\ Chemistry Department, University of Exeter, Exeter EX4 4QD, U.K.
}

(Reçu le 2 avril 1979, accepté le 29 mai 1979)

\begin{abstract}
Résumé. - Les diagrammes de diffraction de rayons $\mathrm{X}$ sur des échantillons très bien orientés de plusieurs phases smectiques B mettent en évidence l'existence de plusieurs types de corrélations entre les couches smectiques. Les couches hexagonales peuvent s'organiser suivant l'ordre ABA... ou ABCA... (A, B et C décrivent la position relative des couches) avec des couches désordonnées de type $A B C$, ou être très peu corrélées entre elles. On n'a pas trouvé de bon exemple d'une structure simple monocouche de type AA...

Abstract. - X-ray diffraction results are presented on very well aligned samples of several smectic B phases which show the existence of several kinds of interlayer correlations. The hexagonal closepacked layers may be stacked with ordered $\mathrm{ABA} \ldots$ or $\mathrm{ABCA} \ldots$ arrangements (where $\mathrm{A}, \mathrm{B}$ and $\mathrm{C}$ denote the relative position of the layers), with a random array of $A B C$-type planes, or with almost no correlation between layers. No clear examples of the simple monolayer packing AA... have been found.
\end{abstract}

This note is concerned principally with the uniaxial ordered smectic phase in which the average arrangement of molecules in the layers is hexagonal close packing and their long axes are approximately orthogonal to the layers. There is no ambiguity about the nomenclature $S_{B}$ for such phases which have hexagonal symmetry [1] (e.g. the $S-3$ phases of $\mathrm{C}_{n} \mathrm{H}_{2 n+1} \mathrm{OPhCHNPh} \mathrm{C}_{m} \mathrm{H}_{2 m+1}[n \mathrm{O} . m]$ compounds) [2]. However, the same name has often been used for the phase of average monoclinic symmetry in which the (pseudo) hexagonal packing is significantly tilted (e.g. $\left.\sim 25^{\circ}\right)$ relative to the layer normal [1] (e.g. the S-V phase of TBBA [3]). Following the recent statement of the nomenclature problem for tilted smectics by Goodby and Gray [4] and the recognition that $S_{B}$ and $S_{H}$ are indeed distinct phases, we follow their recommendation of calling the tilted phase $S_{H}$.

From X-ray diffraction measurements on specimens prepared by careful melting of single crystals it has been established that for both $S_{B}$ and $S_{H}$ phases single domain specimens may be prepared in which the hexagonal or monoclinic symmetry extends throughout the bulk sample [1]. This implies a finite yield stress for the sliding of layers relative to each other and also some long range interlayer correlation. The nature of this correlation has been established for a number of $S_{H}$ phases (notably $40.2[1,2,5]$ and other $n \mathrm{O} . \mathrm{m}$ compounds [2]) by X-ray measurements both on monodomain samples and on powders.
It is clear from the observed $h k l$ reflections that there exists an average monoclinic unit cell with $\mathbf{c} \approx l$ where $l$ is the molecular length. Adjacent layers are thus related by a translation of $\mathbf{c}$ in the direction of the long molecular axis and there exists definite three dimensional order. The same is also true of $S_{G}$ phases. What remains to be determined for particular compounds is a quantitative description of the extend of the order and the nature of the disorder.

For the $S_{B}$ structures no such positive information has been established about the interlayer correlation. The possibility of two extreme cases of extensive and of no interlayer correlations has long been recognized [6,7] but little direct evidence exists. The proven existence in several cases of a hexagonal lattice extending throughout the bulk sample implies at least a long range correlation of the orientation of the layers.

We report here the results of X-ray diffraction experiments on a variety of $S_{B}$ compounds in which extremely well aligned samples were obtained mostly by slow cooling from the isotropic liquid phase in a magnetic field of $>2 \mathrm{~T}$. Such samples have been shown to be almost randomly disordered about the field direction - which for these phases is identical with the molecular long axis (m) and the layer normal $(\langle 00 l\rangle, \mathbf{c}$ or $z)$. This means that all of the reciprocal lattice (for $l$ not too large) is observed in a simple 
Table I. - Type of diffraction pattern shown by some $\mathrm{S}_{\mathbf{B}}$ compounds.

\author{
Compound \\ $\mathrm{C}_{n} \mathrm{H}_{2 n+1} \mathrm{OPhCHNPhC}_{m} \mathrm{H}_{2 m+1}$ \\ $\mathrm{PhPhCHNPhCHCHCOOCH}{ }_{2} \mathrm{CH}\left(\mathrm{CH}_{3}\right)_{2}$ \\ $\mathrm{PhPhCHNPhCHCHCOOC}_{4} \mathrm{H}_{9}$ \\ $\mathrm{C}_{6} \mathrm{H}_{13} \mathrm{OPhCHNPhPh}$ \\ $\mathrm{C}_{8} \mathrm{H}_{17} \mathrm{OPhPhCOOPhOC}{ }_{5} \mathrm{H}_{11}$ \\ $\mathrm{C}_{8} \mathrm{H}_{17} \mathrm{PhPhCOOPhC} \mathrm{H}_{8} \mathrm{H}_{17}$ \\ $\mathrm{C}_{8} \mathrm{H}_{17} \mathrm{OPhPhCOOPhOC}{ }_{8} \mathrm{H}_{17}$ \\ $\mathrm{C}_{5} \mathrm{H}_{11} \mathrm{OPhCHNPhPh}$ \\ $\mathrm{C}_{16} \mathrm{H}_{33} \mathrm{OPhCHNPhPh}$
}

Code

$n \mathrm{O} . \mathrm{m}$

$(40.8 ; 50.7$;

$70.5 ; 60.4$;

$70.7 ; 50.6)$

\begin{tabular}{|c|c|}
\hline IBPBAC & $\mathrm{I}-\mathrm{N}-\mathrm{S}_{\mathrm{A}}-\mathrm{S}_{\mathrm{B}}-\mathrm{S}_{\mathrm{E}}-\mathrm{C}$ \\
\hline BPBAC & $\mathrm{I}-\mathrm{S}_{\mathrm{A}}-\mathrm{S}_{\mathrm{B}}-\mathrm{S}_{\mathrm{E}}-\mathrm{C}$ \\
\hline 60.Ph & $\mathrm{I}-\mathrm{N}-\mathrm{S}_{\mathrm{A}}-\mathrm{S}_{\mathrm{B}}-\mathrm{C}$ \\
\hline 80.05 & $\mathrm{I}-\mathrm{N}-\mathrm{S}_{\mathrm{A}}-\mathrm{S}_{\mathrm{C}}-\mathrm{S}_{\mathrm{B}}-\mathrm{C}$ \\
\hline 8.8 & $\mathrm{I}-\mathrm{S}_{\mathrm{A}}-\mathrm{S}_{\mathrm{B}}-\mathrm{C}$ \\
\hline 80.08 & $\mathrm{I}-\mathrm{N}-\mathrm{S}_{\mathrm{A}}-\mathrm{S}_{\mathrm{C}}-\mathrm{S}_{\mathrm{B}}-\mathrm{C}$ \\
\hline $50 . \mathrm{Ph}$ & $\mathrm{I}-\mathrm{N}-\mathrm{S}_{\mathrm{B}}-\mathrm{C}$ \\
\hline 16O.Ph & $\mathrm{I}-\mathrm{S}_{\mathrm{A}}-\mathrm{S}_{\mathrm{B}}-\mathrm{C}$ \\
\hline
\end{tabular}

Phase sequence

$\mathrm{I}-\mathrm{N}-\mathrm{S}_{\mathrm{A}}-\mathrm{S}_{\mathrm{C}}-\mathrm{S}_{\mathrm{B}}-\left(\mathrm{S}_{\mathrm{H}}\right)-\mathrm{C}$

(see refs. $[2,10])$

Diffraction profile along $\langle 00 l\rangle$, see figure 2

2(e)

(sometimes 2(c), once (6O.4) 2(f))
$2(\mathrm{~d})(+2(\mathrm{e})) ;(2(\mathrm{c}))$ 2(c)
$2(\mathrm{~d})(+2(\mathrm{e}))$
2(c); 2(b)

Failed to prepare aligned sample

2(b) but sample not well aligned

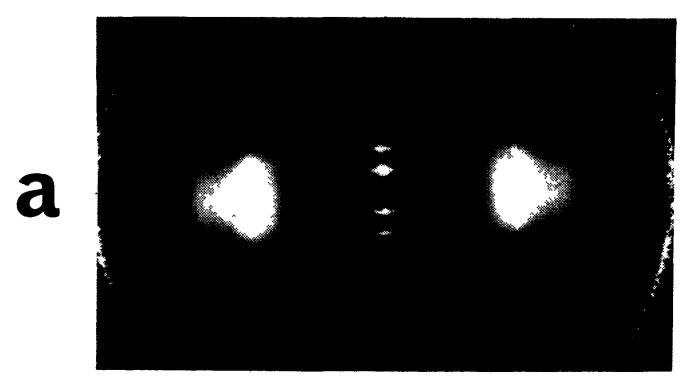

flat plate experiment with fixed sample. Measurements were made photographically using graphite-monochromated $\mathrm{CuK}_{\alpha}$ radiation. An experiment was performed on a sample of 40.8 aligned by surface treatment using a rubbed film of polyvinyl alcohol which gave identical results to magnetically aligned samples. Some experiments were also made on specimens obtained by careful melting of single crystals; these were generally less well ordered (except for the uniaxial disorder) than the magnetically-aligned specimens but enabled us to confirm the hexagonal symmetry of the phase. The liquid crystals investigated together with the type of diffraction pattern observed are summarized in table I.

In all cases the gross features of the diffraction pattern with the X-ray beam parallel to the layers were as shown in the photographs of figure 1 . Two or more orders of the $00 \mathrm{l}$ reflections are observed and within the experimental resolution these are delta functions, implying a crystal-like structure [7]. There is also a strong bar of scattering associated with the reciprocal lattice points $100, \overline{1} 00,010,0 \overline{1} 0, \overline{1} 10$ and $1 \overline{10}$ (hexagonal indexing) and of extent $\sim \pm \mathbf{c}^{*}$. Very weak reflections of type $110, \overline{1} 20$, etc. are also found but the second order reflections (200, etc.) have not been observed (Fig. 1). Strong diffuse scattering is also observed. In many cases, however, with shorter exposure times and provided that the sample is very well aligned the bars of scattering associated with the first hexagonal ring of the reciprocal lattice showed pronounced structure. An example of such a photograph is shown in figure 1 and intensity profiles along the $\langle 00 l\rangle$ rows for a variety of specimens obtained from photographs like figure 1 using a low resolution home-made microdensitometer are shown in figure 2. Profile (d)
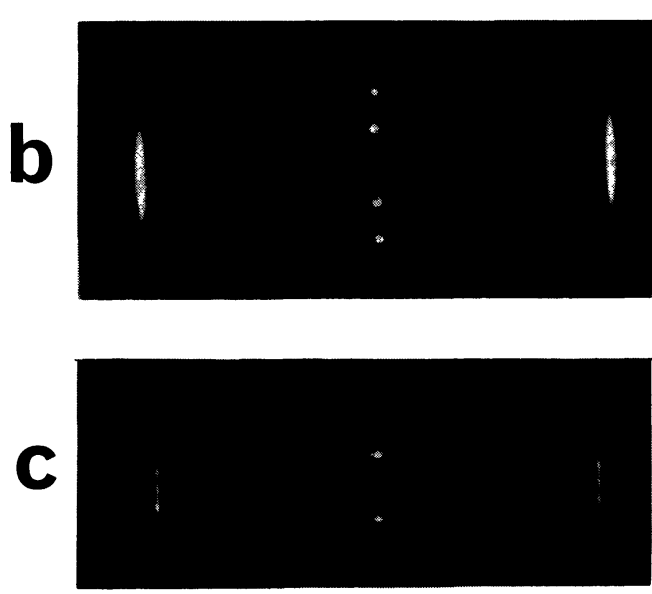

Fig. 1. - X-ray diffraction photographs of $S_{B}$ phases with the beam parallel to the smectic layers. (a) IBPBAC; long exposure which shows the extensive diffuse scattering, $00 \mathrm{l}$ reflections up to $l=3$ and the 110 reflections. The strong outer arcs are container scattering. (b) IBPBAC; shorter exposure, to show the bars of scattering associated with the first $(h k)$ ring of the reciprocal lattice $(100,1 \overline{1} 0$, etc.). (c) 40.8 which gives the clearest example of the structure of the bars of scattering in the first $(h k)$ ring. The microdensitometer profile along such an $\langle 00 l\rangle$ row is shown in figure 2(e).

for IBPBAC was the first observed [8] and an explanation was suggested in which the structure arose 


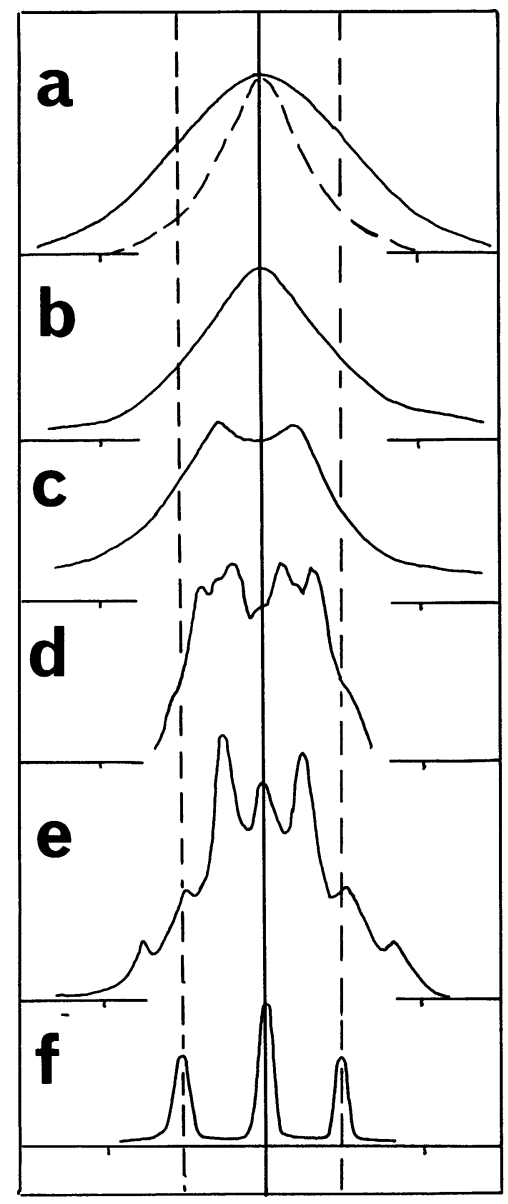

Fig. 2. - Intensity profiles along $\langle 00 l\rangle$ for the first $(h k)$ ring of the reciprocal lattice for $S_{B}$ phases. (a) The Debye-Waller factor $\exp -Q^{2}\left\langle z^{2}\right\rangle$ for $\left\langle z^{2}\right\rangle^{1 / 2} / d=0.1$ (full line) and $\left\langle z^{2}\right\rangle^{1 / 2} / d=0.2$ (dashed line). (b) Experimental result for 8.8 showing uncorrelated layers. (c) Experimental result for IBPBAC sample prepared from a single crystal showing random $A B C$ type layers. (d) Experimental result for IBPBAC sample prepared by cooling in a $2 \mathrm{~T}$ field showing predominantly ABCA... packing plus some ABA... packing. (e) Experimental result for 40.8 showing ABA... packing. (f) Schematic intensity profile expected for simple AA... packing.

from a local tilt of $\sim 5^{\circ}$ of the hexagonally packed molecules about an axis joining opposite corners of a hexagon, which results in the diffraction spots for the uniaxially disordered hexagonal reciprocal lattice appearing at two levels, both displaced from the zero $(l=0)$ layer. The average uniaxial properties were accounted for by a short range interlayer correlation for the tilt direction. A similar type of diffraction pattern and explanation has been reported by Benattar et al. [9] on some compounds of the type $\mathrm{PhPhCH}=\mathrm{NPhC}_{n} \mathrm{H}_{2 n+1}$. Subsequently, however, the results shown in figures 1 and 2 have been accumulated and it has been shown elsewhere [10] that the profile type $2 \mathrm{e}$ observed for essentially all of the $n \mathrm{O} . \mathrm{m}$ series studied by us so far which could be well aligned ( $>5$ examples) are consistent both in symmetry and intensity with an $S_{B}$ phase which is a bilayer structure with an $\mathrm{ABAB}$ type packing of the molecules in which $\mathrm{A}$ is at $(0)$ and $\mathrm{B}$ at
$(2 \mathbf{a} / 3+\mathbf{b} / 3)$, where $\mathbf{a}$ and $\mathbf{b}$ are the hexagonal unit cell vectors of the A layer. This leads to a simple consistent explanation for all of the results so far obtained in terms of the possible stacking arrangements of the hexagonal layers and we believe that our earlier description of the $S_{B}$ structure of IBPBAC (although still internally consistent in this case) is not the correct one. Furthermore for the $n \mathrm{O} . m$ compounds 7 spots are observed along the $\langle 00 l\rangle$ row for the first ring of the reciprocal lattice (i.e. at $l= \pm 3 / 2, \pm 1, \pm 1 / 2$ and 0$)$ and the position of these spots cannot be explained in terms of a slightly tilted monolayer structure. It is of course not contradictory to our conclusions if the compound studied by Benattar et al. was a truly tilted phase with small tilt angle but it should then be called a $S_{H}$ phase.

The intensity of scattering from the $S_{B}$ phases may be written quite generally as

$$
I(h k l) \sim F_{\mathrm{c}}^{2}(h k l)\left\langle F_{\mathrm{m}}(h k l)\right\rangle^{2}
$$

where $F_{\mathrm{c}}(h k l)$ denotes the structure factor for the average molecular centres (the lattice points) including any interlayer disorder while $\left\langle F_{\mathrm{m}}(h k l)\right\rangle$ is the structure factor for the average molecule associated with each point.

If we separate out the important contribution of disorder to $\left\langle F_{\mathrm{m}}(h k l)\right\rangle$, this may be written

$$
\begin{aligned}
\left\langle F_{\mathrm{m}}(h k l)\right\rangle \sim\langle & \left.F_{\mathrm{m}}^{1}(h k l)\right\rangle \times \\
& \times \exp -\left[\left(\frac{2 \pi}{d_{00 l}}\right)^{2} \frac{\langle z\rangle^{2}}{2}\right] f(h x, k y)
\end{aligned}
$$

where the various components of disorder are assumed to be independent and $z$ is the component of molecular displacement perpendicular to the layers (assumed Gaussian) and $f(h x, k y)$ is a disorder function arising from rigid molecule displacement within the layer, and everything else (i.e. the head to tail disorder and any non-uniform atomic displacements) is included in $F_{\mathrm{m}}^{1}(h k l)$. Calculation of $F_{\mathrm{m}}^{1}(h k l)$ requires a detailed model of the molecular conformation and packing but it will generally be a relatively slowly varying function of $Q$ at least in comparison with the very rapid decrease with $Q$ arising from the disorder and to a first approximation we assume it to be constant over the restricted $Q$-range in question (i.e. along the $\langle 00 l\rangle$ rows for $|l| \widetilde{\gtrless}$ ) $\left\langle z^{2}\right\rangle$ may then be obtained from the intensities of the $(00 l)$ reflections and for $S_{B}$ phases relatively few orders are observed $\left(l_{\max } \sim 2\right.$ to 5$)$ so that generally $\left\langle z^{2}\right\rangle^{1 / 2} / d ऽ 0.1$. $f(h x, k y)$ will include the six fold orientational disorder of molecular orientation about $m$ together with the large librational amplitude and lateral displacements.

The envelope of the Debye-Waller factor $\exp \left[-Q^{2}\left\langle z^{2}\right\rangle\right]$ for typical values of $\left\langle z^{2}\right\rangle$ for $S_{B}$ compounds is shown in figure 2. The structure factors $F_{\mathrm{c}}^{2}(h k l)$ may readily be determined for various 
types of packing of the hexagonal layers, both ordered and disordered. This leads to a straightforward interpretation of the main features of results like those shown in figure 2. There are five simple cases, all of which give sharp $00 l$ reflections but are distinguished by their scattering profiles along $\langle 00 l\rangle$ for $h-k \neq 3 n$ [11]. For convenience of comparison the coefficient $l$ will be defined in relation to the layer spacing $\mathbf{d}$ rather than the unit cell length $\mathbf{c}$ so that the observed $00 l$ reflections in all cases (monolayer, bilayer, etc.) are $l=1,2, \ldots$ The five cases are as follows :

1. A simple monolayer (AAA...) packing with adjacent layers related by a simple translation of $d$ along c. The resultant diffraction profile expected along $\langle 00 l\rangle$ rows for $h$ and/or $k \neq 0$ is shown schematically in figure $2(f)$; except for one poorly defined example observed for 60.4 , we have never seen such a profile, which is not surprising as such a stacking configuration must be unstable relative to those below.

2. A simple bilayer (ABAB) packing with adjacent layers displaced alternately by + and $-(2 \mathbf{a} / 3+\mathbf{b} / 3)$ where $\mathbf{a}$ and $\mathbf{b}$ are the vectors defining the hexagonal unit cell of a layer. This will give diffraction peaks along $\langle 00 l\rangle$ for $h-k \neq 3 n$ with relative intensities 3 at $l=p+1 / 2$ and 1 at $l=p$ where $p$ is an integer. A finite correlation length along $c$ will result in broadening of the peaks and this may arise for example from a finite domain size due to the disorder about c, or from stacking faults. Several of the $n \mathrm{O} . \mathrm{m}$ compounds (Table I) have been shown to possess such a structure and a typical diffraction profile is shown for 40.8 in figure 2(e). Within the rather large uncertainties caused by overlapping peaks and/or a diffuse background arising perhaps from regions of random incommensurate stacking at domain boundaries, the intensities as well as the positions of the peaks are consistent with this packing and with $\left\langle z^{2}\right\rangle^{1 / 2} S^{\prime} \AA$. The correlation length of the ordered regions is $\ulcorner 5$ molecules $(\ulcorner 100 \AA)$.

3. A trilayer $A B C \ldots$ packing with $B$ at $(2 \mathbf{a} / 3+b / 3)$ and $C$ at $(a / 3+2 b / 3)$ relative to $A$. This will give diffraction peaks along $\langle 00 l\rangle$ of equal intensity at $l=p+1 / 3$ for $h-k=3 n+1$ and at $l=p-1 / 3$ for $h-k=3 n-1$ and for a sample which is disordered about $\mathbf{c}$ this will result in diffraction rings of equal intensity at $l=1 / 3,2 / 3 \ldots$ etc. Disorder will again cause the peaks to broaden and in addition stacking and distortion faults cause the peaks to be shifted from their ideal positions.

We have observed two examples where this is the predominant packing arrangement (Table I) and the diffraction profile for IBPBAC is shown in figure 2(d). The intensities of the peaks at $l= \pm 1 / 3$ and $\pm 2 / 3$ are also approximately consistent with this packing and $\left\langle z^{2}\right\rangle^{1 / 2} \sim 2.5 \AA$ although the decrease in inten- sity at higher $l$ indicates some additional disorder. In neither of the two examples, however, is the packing purely of $A B C \ldots$ type and the profile of figure 2(d) shows evidence of regions of $A B A B$ packing and possibly considerable incommensurate disorder as well.

4. Random packing of $\mathrm{ABC}$-type layers : this will give a diffuse scattering profile along $\langle 00 l\rangle$ at $h-k \neq 3 n$ with maxima at $l=n+1 / 2$ of intensity 9 relative to minima of intensity 1 at $l=n$. A number of compounds have been found to show a diffraction profile like that in figure 2(c) which approximates to this behaviour except that the minimum at $l=0$ is very shallow. This is probably a result of additional diffuse scattering from incommensurately disordered regions (see below). Only BPBAC so far has been found to show this pattern exclusively (see Table I) but it has been found additionally for IBPBAC samples prepared from single crystals via the $\mathrm{S}_{\mathrm{E}}$ phase and for several $n \mathrm{O} . m$ compounds prepared by cooling in a field. In both cases the preparation conditions presumably did not allow formation of the more stable $\mathrm{ABC}$ or $\mathrm{ABAB}$ packings.

5. No correlation between layers : the intensity along $\langle 00 l\rangle$ for all rows except $h=k=0$ will be a structureless diffuse bar of length about $2 / d$. The profile shown in figure 2(b) is typical of those observed for a number of compounds. It is roughly Gaussian in shape with a full width at half height of $2 / d$ which implies a correlation length of only about one layer thickness. This type of diffraction profile might also be considered as arising from, say, an ABAB packing with a sufficiently low correlation length to broaden the profile of 2(e) to give that of 2(b). This requires a correlation length of less than about two layers so that in any case a profile like that of 2(b) must mean that a, b correlations between the smectic layers do not extend beyond adjacent layers.

This type of pattern is the one observed most frequently (Table I) but it does not necessarily imply that a more ordered packing cannot be produced - for example by long annealing.

All samples which are completely disordered around c must have some incommensurately disordered layers at the boundaries between domains of differing orientation and hence their scattering along $\langle 00 l\rangle$ except for $h=k=0$ must have a diffuse component of type 2(b).

The above results make it clear that many $S_{B}$ phases have a regular ordered packing arrangement of the hexagonal layers giving a three dimensional crystalline structure. However the energy minima for the relative location of the layers are in general shallow so that disorder is very easily introduced and may be enough to give essentially uncorrelated layers. The relationship between the extent and type 
of interlayer correlation and the chemical constitution remains to be established.

A feature of all $S_{B}$ phases studied is the small number of diffraction peaks observed. The rapid decrease in intensity with increasing $l$ means that $\left\langle z^{2}\right\rangle^{1 / 2} / d_{001} ₹ 0.1$ while the decrease with increasing $h, k$ requires a similar value for the reduced rms centre of mass displacement in the plane $\left[\left\langle X^{2}\right\rangle^{1 / 2} / d_{100}\right]$ in addition to the 6 fold orientational disorder. This cannot account however for the much smaller intensity of (200) compared with (110) reflections (the former are in fact not observed). This is associated with disorder in the stacking of the hexagonal layers since faults in the regular packing cause the reciprocal lattice nodes for $h-k \neq 3 n$ (e.g. 200 but not 110) to broaden and consequently to appear weaker.

We have established for the first time the nature of the interlayer correlations in a number of $S_{B}$ phases. It would now be useful to extend these measurements to a wider variety of compounds in order to develop an understanding of the causes underlying the occurrence of particular structures. To this end it will also be necessary to develop further the art of preparing very well aligned (and preferably true monodomain) samples and to study them by accurate intensity measurements with higher resolution than has generally been used previously. We are now beginning this work.

\section{References}

[1] Levelut, A. M., Doucet, J. and Lambert, M., J. Physique 35 (1974) 773

[2] Doucet, J. and Levelut, A. M., J. Physique 38 (1977) 1163.

[3] Doucet, J., Levelut, A. M. and Lambert, M., Phys. Rev. Letts. 32 (1974) 201.

[4] Goodby, J. W. and Gray, G. W., Mol. Cryst. Liq. Cryst. Lett., in press.

[5] De Vries, A. and Fischel, D. L., Mol. Cryst. Liq. Cryst. 16 (1972) 311.

[6] De VRIES, A., Chem. Phys. Letts. 28 (1974) 252.
[7] De Gennes, P. G. and Sarma, G., Phys. Lett. 38A (1972) 219 ; De Gennes, P. G., Mol. Cryst. Liq. Cryst. 21 (1973) 49.

[8] Richardson, R. M., Leadbetter, A. J. and Frost, J. C., Ann. Phys. 3 (1978) 177.

[9] Benattar, J. J., Levelut, A. M. and Strzelecki, L., J. Physique 39 (1978) 1233.

[10] Leadbetter, A. J., Kelly, B. A., Mazid, M. A., Goodby, J. W. and Gray, G. W., Submitted to Phys. Rev. Letts.

[11] GuINIER, A., X-ray Diffraction (W. H. Freeman) 1963. 